\title{
Surveying physics and astronomy students' attitudes and approaches to problem solving
}

\author{
Andrew Mason, ${ }^{1}$ Melanie Good, ${ }^{2}$ and Chandralekha Singh ${ }^{2}$ \\ ${ }^{1}$ Department of Physics and Astronomy, University of Central Arkansas, Conway, AR 72035 \\ ${ }^{2}$ Department of Physics and Astronomy, University of Pittsburgh, Pittsburgh, PA 15260
}

\begin{abstract}
We examined how introductory physics students' attitudes and approaches to problem solving compare to those of introductory astronomy students', using a previously validated survey, the Attitudes and Approaches to Problem Solving (AAPS) survey. In addition, we compared the performance of the introductory physics and astronomy students on the factors which were identified in a factor analysis in the original validation study. We found that introductory astronomy students' overall average AAPS score was significantly more favorable than that of introductory physics students $(p<0.01)$, and the effect size was large (Cohen's $d=0.81)$. We also found that introductory astronomy students' scores were more favorable in all clusters of questions except for one. We also found that introductory physics and astronomy students were equally capable of solving two isomorphic problems posed to them, and that the majority of introductory physics and introductory astronomy students reported that the problem posed in the astronomy context was more interesting to them. Interviews suggest that the context of astronomy in problem solving may be more interesting for students and could be one possible explanation for the more favorable AAPS scores amongst introductory astronomy students.
\end{abstract}

\section{INTRODUCTION}

Differences between introductory physics and introductory astronomy classes and students: A typical calculusbased or algebra-based introductory physics course may offer a different experience for students compared to a typical introductory astronomy course for science and engineering majors. Introductory physics is mandatory for most science and engineering majors but introductory astronomy, while mandatory for some students who take it as part of the "physics and astronomy" major, is an elective for science or engineering majors. Moreover, although the difficulty level of these courses is comparable and the courses cover similar physics principles underlying the relevant content, the content of the two courses often contain very different themes.

While requiring the same level of rigor, these classes are often framed differently to students. From the manner in which an introductory astronomy course for physics and astronomy majors is organized, it is clear that the focus is on understanding the large-scale structure of the universe and the rules that govern the past and future evolution of astrophysical objects. In an introductory physics course, the focus is often on helping students learn to apply the laws of physics in simplified contexts, e.g., applying Newton's laws to blocks on inclined planes or masses connected via ropes and pulleys. Also, astronomy classes often involve the use of many photographic images of objects in space, whereas physics classes often rely upon sketches, cartoons, or other abstract representations of the objects being studied.

Assessing introductory physics students' views using attitudinal surveys: Understanding students' attitudes and approaches to problem solving is important because it can have instructional implications [1]. Many attitudinal surveys have been developed to assess students' beliefs about physics, e.g., those in Refs. [2]-4]. The Attitudes and Approaches to Problem Solving (AAPS) survey is a modified version of the Attitudes to Problem Solving Survey [3, 4] (which in turn is based upon MPEX survey in Ref. [2]), and was developed to include questions about approaches to problem solving [5, 6]. The focus of the research presented here is on analyzing, compar- ing, and interpreting AAPS survey responses for introductory algebra-based and calculus-based physics and introductory astronomy students as a posttest at the end of the course (the survey was not given as a pretest due to time-constraints). This survey was validated earlier based upon faculty, graduate and introductory student responses [5, 6]. During validation, expected "favorable" responses agreed with faculty responses to a high degree, and it was found to be the case that, on average, introductory students responded differently from graduate students and faculty, in that introductory student responses were found to be less "favorable", on average, compared to graduate students and faculty [5].

Research questions: Our two tightly-related research questions in the research presented here are:

RQ1. How do the average overall scores on the AAPS survey questions and on force concepts compare for introductory astronomy students and introductory physics students and how do their AAPS responses compare to expert responses?

RQ2. How do scores on specific clusters of questions on the AAPS survey compare for introductory astronomy students and introductory physics students?

\section{METHODOLOGY}

Courses and participants: The courses in our study consisted of both algebra-based and calculus-based introductory physics, and introductory astronomy for science and engineering majors at the University of Pittsburgh. The introductory astronomy course discussed here is intended primarily for science and engineering majors, and is a required part of the curriculum for undergraduate students who are majoring in "physics and astronomy". The algebra-based introductory physics classes are required for some science majors, such as biology and neuroscience. The calculus-based physics classes are required for engineering and physics majors. A total of 606 students participated in this study, including 541 introductory physics students (including algebra-based and calculusbased courses) and 65 introductory astronomy students. The AAPS survey responses from graduate students and faculty are included in order to serve as a benchmark of more expertlike responses [5]. A total of 42 graduate students and 12 fac- 
ulty responses to the AAPS survey are included. A subset of the total sample of students participated in individual followup interviews, each lasting approximately 1 hour. Those interviewed included 12 introductory physics students and 8 introductory astronomy students.

Data collection: The AAPS survey was given to students in this study near the end of the semester. The items in the AAPS survey are statements which can elicit agreement or disagreement, and responses are given on a 5 point Likert scale. Once completed, spreadsheets of the results were produced and scores for each question were computed by assigning +1 to a favorable response, -1 to an unfavorable response, and 0 to a neutral response, which we adopted for consistency with the convention used when the AAPS survey was originally validated [5]. We also computed the percentage of the total responses that were favorable, neutral, and unfavorable.

In order to gather qualitative data, the written survey data collection was followed by individual hour-long interviews with introductory physics and introductory astronomy students. The interviews utilized a semi-structured "think aloud" protocol in which students first answered the AAPS survey questions along with providing reasoning for their answers on their own. Follow up questions were then asked for clarification of points not made clear. In addition, in the interviews, both introductory physics and introductory astronomy students were presented with an isomorphic pair of problems. They were first asked to solve both problems and then were asked about which problem was more difficult or enjoyable. Both problems required the students to solve for the speed of an object based upon the assumption of uniform circular motion with centripetal acceleration (one context was the motion of a yo-yo and another was Earth's motion around the Sun). Thus, the problems required the same concepts and formulas, but contained different contexts-the astronomy problem involved the motion of the Earth around the Sun and the physics problem involved the circular motion of a yo-yo whirled in a horizontal circle. The purpose was to determine if physics and astronomy students were equally proficient in solving these problems, whether one of the problems was more challenging than the other and whether there was something about the context of astronomy or physics that produced different attitudes or approaches to the problem-solving process. We also used scores on the Force Concept Inventory (FCI) [7] to investigate conceptual performance of physics and astronomy students.

\section{RESULTS}

RQ1. How do the average overall scores on the AAPS survey questions and on force concepts compare for introductory astronomy students and introductory physics students and how do their AAPS responses compare to expert responses? Figure 1 shows that introductory physics and astronomy students had less expertlike attitudes and approaches to problem solving compared with graduate students and faculty [5]. However, astronomy students had more expertlike attitudes and approaches than physics students. All differences in overall scores were found to be statistically significant, with $p<0.001$ and effect sizes with Cohen's d [8] of $d>0.8$.

As noted, while the content is presented differently in

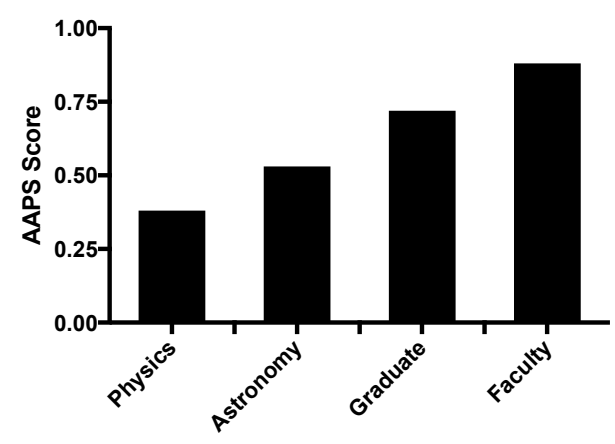

FIG. 1: Average AAPS scores of introductory physics students, introductory astronomy students, graduate students, and faculty.

introductory physics and astronomy classes, the underlying physics principles and level of rigor involved in the instruction are equivalent. Also, while the courses are presented very differently, foundational topics in physics, such as forces and energy are part of the content of both courses. Indeed, when we compare the performance on the Force Concept Inventory (FCI), we find that students in introductory astronomy had very similar average score on the pre-test $(47 \%$ for physics and $46 \%$ for astronomy) and post-test (58\% for physics and $62 \%$ for astronomy). Moreover, at the end of the interviews, introductory astronomy and physics students were presented with a pair of isomorphic problems (one written in and one without the context of astronomy). Both problems required the use of the concept of centripetal force and the assumption of a uniform circular motion in order to solve for the speed. We find that $75 \%$ of introductory physics students and $86 \%$ of introductory astronomy students successfully solved both problems, with additional students in both groups who solved a significant fraction of both isomorphic problems correctly but made some mistakes along the way. Like the FCI results which show similar conceptual performance, this similarity in performance on the isomorphic problems suggests that student performance on quantitative problem solving is also comparable. We note that while performance of students appears to be similar on the isomorphic problems, the majority of both introductory physics and astronomy students reported that they found the astronomy context more interesting, even if they found it to be somewhat more difficult to solve or challenging to think about due to the large numerical values involved in the astronomy problem.

Considering the students' comparable facility at both the FCI and the isomorophic problem solving, the difference in attitudes and approaches to problem solving in physics and astronomy is not likely to be due to the differences in rigor of treatment. Instead, there may be something about the manner and context in which introductory astronomy classes are taught which might at least partly account for this difference. In other words, while introductory astronomy students learned just as many physics concepts pertaining to forces and Newton's laws as introductory physics students, as measured by the FCI, introductory astronomy students' more favorable attitudes than introductory physics students may be due to a more engaging context in which the content is learned and the manner in which the entire course curriculum is framed. 
The fact that most students preferred the astronomy context when solving the isomorphic problems appears to support this explanation. For example, one astronomy student in an interview said (about the astronomy problem) "That's cool to me because then you could think about well what if all of a sudden we stop orbiting and we go off on a tangent, and at that speed, well that sucks, that's going to be rough." On the other hand he indicated that the non-astronomy problem was not as captivating or interesting, stating that if the yo-yo string broke, "that just means it's hitting my TV and I'm getting yelled at."

Like this student, many student responses during interviews suggested a lack of engagement with the context in which physics is often presented in problems and in the classroom. For example, one student expressed that he would enjoy physics classes more if he could relate them to something interesting or realistic. He went on to point out a common type of introductory physics problem as a counter-example to an interesting or realistic type of problem, stating "How many times are you realistically doing that with a pulley?" Similarly, a student who had taken both introductory physics and astronomy expressed excitement when comparing the two experiences, stating: "Astronomy is more exciting. I just read this story today about a galaxy that doesn't have any dark matter. That's freaking awesome... Physics doesn't do that."

RQ2. How do scores on specific clusters of questions on the AAPS survey compare for introductory astronomy students and introductory physics students? There are a few AAPS survey questions in which physics students outperformed astronomy students. However, for most of the AAPS survey questions, astronomy students outperformed physics students. A factor analysis was performed earlier in the AAPS validation study [5]. Table I] compares the factors identified in the validation study [5] with responses from introductory physics and astronomy students. Astronomy students generally outperformed physics students in all factors except for Factor 2, which is related to drawings and scratchwork while problem solving.

Among the AAPS survey questions on which introductory astronomy students outperformed introductory physics students, many of the questions center around Factor 1, which is related to metacognition and enjoyment in problem solving. In fact, Factor 1 accounted for more of the variance than any other factor in Ref. [5]. For example, question 27 in Factor 1 (see Table 1) states, "I enjoy physics/astronomy even though it can be challenging at times." (Note that physics students only saw the word "physics" in this question and astronomy students only saw the word "astronomy"). While $72 \%$ of astronomy students agreed with this statement, only $40 \%$ of physics students agreed with it.

Some questions in Factor 1 asked about reflection and conceptual thinking over "plug-and-chug" approaches. For example, question 4 within Factor 1 states "In solving problems in physics/astronomy, I always identify the physics/astronomy principles involved in the problem first before looking for corresponding equations." A favorable response to this question would be to agree with the statement, as $80 \%$ of the introductory astronomy students did. Fewer introductory physics
TABLE I: Average AAPS survey scores of physics and astronomy students by question and factors identified in Refs. [5, 6]. More favorable scores appear in boldface. The order of AAPS survey problem numbers within each factor reflects the relative "loading" of each question within each factor in Refs. [5, 6].

\begin{tabular}{|c|c|c|c|}
\hline & $\begin{array}{l}\text { Question } \\
\text { Number }\end{array}$ & $\begin{array}{l}\text { Physics } \\
\text { Score }\end{array}$ & $\begin{array}{l}\text { Astronomy } \\
\text { Score }\end{array}$ \\
\hline \multirow{12}{*}{$\begin{array}{l}\text { Factor } 1 \text { (Metacognition and } \\
\text { enjoyment of problem-solving) }\end{array}$} & 13 & 0.56 & $\underline{0.75}$ \\
\hline & 14 & 0.32 & $\underline{0.72}$ \\
\hline & 7 & 0.61 & $\underline{0.83}$ \\
\hline & 10 & 0.58 & $\underline{0.88}$ \\
\hline & 22 & 0.52 & $\underline{0.69}$ \\
\hline & 29 & 0.74 & $\underline{0.82}$ \\
\hline & 20 & -0.19 & $\underline{0.26}$ \\
\hline & 4 & 0.41 & $\underline{0.71}$ \\
\hline & 25 & 0.56 & $\underline{0.71}$ \\
\hline & 27 & 0.03 & $\underline{0.65}$ \\
\hline & 6 & 0.24 & $\underline{0.51}$ \\
\hline & 21 & 0.71 & $\underline{0.86}$ \\
\hline \multirow{4}{*}{$\begin{array}{l}\text { Factor } 2 \text { (Use of drawings and scratch- } \\
\text { work) }\end{array}$} & 18 & $\underline{0.69}$ & 0.48 \\
\hline & 17 & $\underline{0.55}$ & 0.28 \\
\hline & 15 & $\underline{0.74}$ & 0.51 \\
\hline & 19 & 0.77 & 0.86 \\
\hline \multirow{6}{*}{$\begin{array}{c}\text { Factor } 3 \text { (Perception of problem- } \\
\text { solving approach) }\end{array}$} & 5 & 0.16 & $\underline{0.23}$ \\
\hline & 11 & -0.03 & $\underline{0.26}$ \\
\hline & 12 & -0.06 & $\underline{0.13}$ \\
\hline & 8 & 0.67 & 0.66 \\
\hline & 26 & 0.37 & $\underline{0.60}$ \\
\hline & 9 & 0.24 & $\underline{0.31}$ \\
\hline \multirow{5}{*}{$\begin{array}{l}\text { Factor } 4 \text { (General expert-novice } \\
\text { differences in problem-solving) }\end{array}$} & 8 & 0.67 & 0.66 \\
\hline & 28 & 0.75 & $\underline{0.89}$ \\
\hline & 21 & 0.71 & $\underline{0.86}$ \\
\hline & 24 & $\underline{0.43}$ & 0.15 \\
\hline & 29 & 0.74 & $\underline{0.82}$ \\
\hline \multirow{2}{*}{ Factor 5 (Use of symbolic notation) } & 31 & 0.08 & $\underline{0.12}$ \\
\hline & 30 & 0.70 & $\underline{0.80}$ \\
\hline \multirow{4}{*}{$\begin{array}{c}\text { Factor } 6 \text { (Problem-solving } \\
\text { confidence) }\end{array}$} & 1 & 0.14 & $\underline{0.40}$ \\
\hline & 24 & $\underline{0.43}$ & 0.15 \\
\hline & 23 & 0.40 & $\underline{0.75}$ \\
\hline & 6 & 0.24 & $\underline{0.51}$ \\
\hline \multirow{2}{*}{$\begin{array}{c}\text { Factor } 7 \text { (Solving different problems } \\
\text { using the same principles) }\end{array}$} & 33 & 0.70 & $\underline{0.80}$ \\
\hline & 32 & 0.46 & $\underline{0.63}$ \\
\hline \multirow{3}{*}{ Factor 8 (Sensemaking) } & 16 & 0.23 & $\underline{0.58}$ \\
\hline & 2 & 0.19 & $\underline{0.49}$ \\
\hline & 5 & 0.16 & $\underline{0.23}$ \\
\hline \multirow{4}{*}{$\begin{array}{l}\text { Factor } 9 \text { (Problem-solving } \\
\text { sophistication) }\end{array}$} & 3 & $\underline{0.15}$ & -0.15 \\
\hline & 20 & -0.19 & $\underline{0.26}$ \\
\hline & 25 & 0.56 & $\underline{0.71}$ \\
\hline & 9 & 0.24 & 0.31 \\
\hline
\end{tabular}

students (62\%) responded favorably by comparison.

Factor 6 (see Table 1) was another one for which introductory astronomy students gave more favorable responses than introductory physics students. This factor has to do with problem solving confidence and willingness to persist in working through challenging problems. For example, question 23 states "If I cannot solve a problem in $10 \mathrm{~min}$, I give up on that problem." A favorable response of disagree was given by $82 \%$ 
of astronomy students but only $61 \%$ of physics students.

The only factor in which introductory astronomy students gave less favorable responses compared with physics students was Factor 2 (see Table 1), which is related to the use of drawings and scratchwork while solving problems. Followup interviews suggest one possible reason for less favorable responses. As one student described, certain topics that come up in astronomy problems may be more difficult to depict in a drawing, stating: "For example, involving things like a spectrum...I'm not going to draw a light wave." Other students expressed similar sentiments. Since introductory physics problems often focus more on simpler physical objects that lend themselves well to drawing, this could account for the less favorable responses of astronomy students regarding the use of drawing and scratchwork in problem solving.

\section{DISCUSSION AND CONCLUSION}

Similar students, different attitudes: Our results indicate that on the AAPS survey, introductory astronomy students, overall, had more expertlike attitudes and approaches than introductory physics students, and had more expertlike attitudes on most clusters of questions found via factor analysis (see Table 1). These differences in attitude appear despite the fact that the majority of students in both classes are science majors with comparable performance levels on tests of conceptual understanding (as revealed by average FCI scores) and problem solving skill (as revealed by isomorphic problem solving).

Captivating content: The differences between AAPS scores of introductory physics and introductory astronomy appear to suggest that there may be something about the subject matter in astronomy that might promote metacognition and may be more interesting and engaging for students compared to what they learn in a typical physics class. For example, it is possible that learning about galaxies and black holes and viewing images of outer space is more captivating than learning about pulleys and inclined planes. One interviewed student described the appeal of real astronomical images compared with images one might encounter in a physics class as follows, "For astronomy there are all these real images... you can't take a picture of a projectile the same way." Likewise, the majority of students who were interviewed indicated that they enjoyed the astronomy problem more than the isomorphic physics problem. If it is the case that students find astronomy and astronomy problems more interesting, it is possible that more captivating instruction of physics classes, including more engaging contexts such as those used in astronomy classes, may improve physics students' attitudes and approaches to problem solving.

Impact of motivation on learning: Researchers have of- ten divided students into a few broad categories based upon their motivational characteristics, e.g., those who are focused mainly on performing well in a class without much focus on achieving deep understanding, i.e., those with a "performance" motivation orientation, or those who are focused on achieving mastery of the material, i.e., a "mastery" motivation orientation [9]. These different extrinsic or intrinsic motivational goals can have a negative or positive feedback loop effect on student attitudes and may shape their problem solving beliefs and processes through the level of engagement and sense-making throughout a course [9].

While the student body is similar in physics and astronomy courses in this investigation, many students take the astronomy course as an elective. This may point to an intrinsic motivation to take this course as opposed to an extrinsic motivation for the physics course, which is required for most students. We note however that while students taking a class as an elective or as a requirement could be expected to be one factor in their motivational characteristics at the end of the course, we find that it is possible to acquire equivalent conceptual learning and problem-solving skills, e.g., in force concepts, in both classes (as is suggested here by astronomy students' FCI scores and facility at solving isomorphic problems, which were equivalent to those of physics students).

Because many AAPS survey questions tap into issues of motivational goals, one hypothesis inspired by our finding is that the more favorable responses in introductory astronomy at the end of the course may imply that not only are students more motivated to learn the astronomy content from the beginning but the content encourages a more mastery-oriented motivational goal and keeps students in a positive feedback loop so that their attitudes remain higher than those in introductory physics at the end of the course. This hypothesis will be tested in a future investigation in which we will administer the AAPS survey both as a pre and posttest to both sets of students. In particular, these findings suggest the possibility that instruction of the mandatory introductory physics classes could potentially be tailored to more positively motivate students without sacrificing rigor in learning if they are appropriately tailored. Since there is a significant overlap in the type of students taking introductory physics and astronomy, and since their performance in conceptual and quantitative measures on force concepts is equivalent, it will be useful to uncover whether the discrepancy in their AAPS scores could at least partly be addressed through the ways in which introductory physics is traditionally taught. In particular, if students in introductory physics can be motivated to learn and more effectively engage with the content, as it appears students in introductory astronomy are, then attitudes and approaches to problem solving in introductory physics may improve.
[1] K. Harper, Phys. Educ. 44, 250 (2006).

[2] E. Redish, J. Saul, R. Steinberg, Am. J. Phys 66, 212 (1998).

[3] K. Cummings, S. Lockwood, and J. Marx, PERC Proc. (2004).

[4] J. Marx and K. Cummings, AIP Conf. Proc. 883, 101 (2007).

[5] A. Mason and C. Singh, Euro. J. Phys. 37, 055704 (2016).
[6] A. Mason and C. Singh, Phys. Rev. STPER 6, 020124 (2010).

[7] D. Hestenes, M. Wells, G. Swackhamer, The Phys. Teach. 30, 141 (1992).

[8] J. Cohen, Statistical Power Analysis, 2nd Ed. Routledge, (1988).

[9] T. J. Nokes-Malach and J. Mestre, Educ. Psych. 48, 184 (2013). 